\title{
Peripheral effector memory regulatory T-cells are incremented and functionally enhanced in successful mite monomeric allergoid sublingual immunotherapy
}

\author{
Claudia Petrarca ${ }^{1}$, Paola Lanuti ${ }^{1}$, Sabrina Di Pillo ${ }^{2}$, Marianna Petrosino ${ }^{2}$, Marco \\ Marchisio $^{1}$, Laura Pierdomenico ${ }^{1}$, Gianni Mistrello ${ }^{3}$, Enrico Compalati ${ }^{3}$, Takemi Otzuki ${ }^{4}$, \\ Roberto Paganelli ${ }^{1}$, and Mario Di Gioacchino ${ }^{1}$ \\ ${ }^{1}$ University Gabriele d'Annunzio of Chieti and Pescara Department of Medicine and Aging \\ Science \\ ${ }^{2}$ Ospedale Clinicizzato SS Annunziata Chieti \\ ${ }^{3}$ Lofarma SpA \\ ${ }^{4}$ Department of Hygiene, Kawasaki Medical School
}

July 13, 2020

\begin{abstract}
Background. Sublingual allergen immunotherapy (SLIT) is recognized as both efficacious and safe, especially when using monomeric allergoids. Its mechanism of action is based on the differentiation, activation, and maturation of allergen-specific Regulatory T-cells (Tregs), fundamental for the activation and maintenance of immune tolerance. Tregs, originally identified as a subpopulation of Foxp3 expressing CD4+CD25high T-cells; however, under inflammatory conditions, they are conveniently identified by the surface antigen CD127 and are subtyped as Resting, Activated and Effector Tregs by surface expression of CD45RA, HLA-DR and CD39 that represent markers of differentiation status, inhibitory and recall potential. Hence, in this study, circulating Tregs were characterized in pediatric patients suffering from allergic rhinitis (AR) treated by SLIT and to verify the possible correlation between some Treg subsets with successful SLIT. Methods. Twenty children suffering from mite AR and/or asthma were enrolled. AR severity was assessed by evaluating ACT and ARIA scores at baseline and after 12months of mite-SLIT. Treg analysis of PBMC pre- and post-SLIT, was based on cytofluorimetric determination of total Tregs, as CD4+CD25highCD39+CD127low/neg, further characterized for CD45RA, HLA-DR and CD39 expression. Results. After SLIT, Resting Tregs were significantly reduced whilst Activated/Effector Tregs CD45RAneg (memory Tregs) resulted increased; moreover, CD39 and HLA-DR expression on Tregs was significantly increased. Notably, the intensity of HLA-DR expression on Tregs positively correlated with the improvement of the clinical scores. Conclusions. Our findings suggest that effective SLIT is associated with re-patterning of the differentiation status of Tregs, particularly with the generation of allergen-specific memory effector Tregs.
\end{abstract}

Peripheral effector memory regulatory T-cells are incremented and functionally enhanced in successful mite monomeric allergoid sublingual immunotherapy

Claudia Petrarca ${ }^{1,2}$, Paola Lanuti ${ }^{1,2}$, Sabrina Di Pillo ${ }^{3}$, Marianna Petrosino ${ }^{3}$, Gianni Mistrello ${ }^{4}$, Enrico Compalati $^{4}$, Takemi Otzuki ${ }^{5}$, Marco Marchisio ${ }^{1,2}$, Laura Pierdomenico ${ }^{1,2}$, Roberto Paganelli ${ }^{1,2}$, Mario Di Gioacchino $^{1,2,6}$

1) Department of Medicine and Science of Aging, G. d'Annunzio University, Chieti, Italy

2) Center for Advanced Science and Technology (CAST), G. d'Annunzio University, Chieti, Italy 
3) Unit of Pediatrics, University Hospital, Chieti, Italy

4) Lofarma Allergeni, SpA, Milan, Italy

5) Department of Hygiene, Kawasaki Medical School, Okayama, Japan

6) Foundation for Clinical Immunotherapy and Advanced Biological Treatments, Pescara, Italy

\section{Corresponding Author}

Claudia Petrarca

Associate Professor of Occupational Medicine

Unit of Nanoimmunotoxicology, Allergy and \& Occupational Biorepository

DMSI-CAST

University "G. d'Annunzio" Chieti-Pescara

Via Luigi Polacchi, 11

66013 Chieti - Italy

phone $+39.871 .541 .290 / 1$

Mail:claudia.petrarca@unich.it

\section{Abstract}

\section{Background}

Sublingual allergen immunotherapy (SLIT) is recognized as both efficacious and safe, especially when using monomeric allergoids. Its mechanism of action is based on the differentiation, activation, and maturation of allergen-specific Regulatory T-cells (Tregs), fundamental for the activation and maintenance of immune tolerance. Tregs, originally identified as a subpopulation of Foxp3 expressing CD4 ${ }^{+} \mathrm{CD} 25^{\text {high }}$ T-cells; however, under inflammatory conditions, they are conveniently identified by the surface antigen CD127 and are subtyped as Resting, Activated and Effector Tregs by surface expression of CD45RA, HLA-DR and CD39 that represent markers of differentiation status, inhibitory and recall potential. Hence, in this study, circulating Tregs were characterized in pediatric patients suffering from allergic rhinitis (AR) treated by SLIT and to verify the possible correlation between some Treg subsets with successful SLIT.

Methods. Twenty children suffering from mite AR and/or asthma were enrolled. AR severity was assessed by evaluating ACT and ARIA scores at baseline and after 12-months of mite-SLIT. Treg analysis of PBMC pre- and post-SLIT, was based on cytofluorimetric determination of total Tregs, as $\mathrm{CD} 4{ }^{+} \mathrm{CD} 25^{\text {high }} \mathrm{CD} 39^{+} \mathrm{CD} 127^{\text {low }}{ }^{\text {neg }}$, further characterized for CD45RA, HLA-DR and CD39 expression.

Results. After SLIT, Resting Tregs were significantly reduced whilst Activated/Effector Tregs CD45RA ${ }^{\text {neg }}$ (memory Tregs) resulted increased; moreover, CD39 and HLA-DR expression on Tregs was significantly increased. Notably, the intensity of HLA-DR expression on Tregs positively correlated with the improvement of the clinical scores.

Conclusions. Our findings suggest that effective SLIT is associated with re-patterning of the differentiation status of Tregs, particularly with the generation of allergen-specific memory effector Tregs.

Keywords : Allergic Rhinitis, Asthma, SLIT, Regulatory T-cells, Resting regulatory T-cells, Activated regulatory T-cells, Effector regulatory T-cells

\section{List of abbreviations:}


AIT, allergy immunotherapy; ACT, asthma control test; AR, allergic rhinitis; ARIA, allergic rhinitis and its impact on asthma; aTreg, activated regulatory T-cell; BDT, bronchodilation test; COPD, chronic obstructive pulmonary disease; DMSO, dimethyl sulfoxide; EDTA, Ethylenediaminetetraacetic acid; eTreg, effector regulatory T-cell; FACS, fluorescence activated cell sorting; FCS, fetal calf serum; FMO, fluorescence minus one; Ig, immunoglobulin; ILC-2, innate lymphoid type 2 cells; MFI, mean fluorescence intensity; PBMC, peripheral blood mononuclear cells; rTreg, resting regulatory T-cell; SLIT, sublingual allergen immunotherapy; Treg, regulatory T-cell; VAS, visual analogue scale;

\section{INTRODUCTION}

Allergen Immunotherapy (AIT) is the only treatment able to modify the natural course of respiratory allergy and is efficacious in allergic rhinitis and asthma [1,2]. Sublingual route of AIT (SLIT) has been recognized as a safe, and effective way of administration, that reduces the risk of adverse reactions (3), particularly suitable for children adherence to the treatment. The use of monomeric allergoids seems to enhance SLIT safety and effectiveness as reported by studied in animal models $(4,5)$ and in humans $(3,6,7)$. The long-term beneficial effects of AIT have been attributed to the generation of immune tolerance to the allergen; the induction of allergen-specific Regulatory T-cells (Tregs) is of crucial importance as they are key regulators of immunological processes in peripheral tolerance to allergens [8] able to suppress inflammatory dendritic cells, innate lymphoid type 2 cells (ILC-2), allergen-specific Th2 effector cells, mast cells, basophils and eosinophils and to induce an $\operatorname{IgE} / \operatorname{IgG} 4$ switch $[9,10]$. Tregs have originally been identified as a subpopulation of $\mathrm{CD} 4{ }^{+} \mathrm{CD} 25^{+}$T-cells expressing the key transcription factor Foxp3 $\left(\mathrm{CD} 4{ }^{+} \mathrm{CD} 25^{\text {high }}\right.$ FoxP $\left.3{ }^{+}\right)$, which is essential for their development and function; in particular, the increment of FoxP3 ${ }^{+}$Tregs and their related cytokines was described to be associated to effective AIT in mouse models $[4,5,11,12]$ and clinical settings $[3,13,14]$. In children with allergic rhinitis (AR), follicular Foxp3 ${ }^{+}$Tregs are defective and shown to be improved by AIT [15].

In humans, FoxP3 ${ }^{+}$Tregs are not functionally homogeneous and their detection is complex and uncertain due to FoxP3 intracellular localization. In its place, the surface antigen CD127, whose level of expression inversely correlates with that of intracellular FoxP3, conveniently allows to discriminate Tregs according to the most recent characterization of human subpopulations described in healthy subjects [16], B-CLL [17], transplant recipients [18], COPD [19], multiple sclerosis [20] and neurologic disorders [21]. Moreover, the expression level of the constitutive CD39 surface antigen can be analyzed as a measure of their inhibitory function [22] and HLA-DR expression level is a monitor of their differentiation status [18] and identifies a functionally distinct profoundly suppressive population [23,24]. Specifically, CD $4^{+} \mathrm{CD} 25^{\text {high }} \mathrm{CD} 39^{+} \mathrm{CD} 127^{\text {neg }}$ cells can be subtyped as Resting (CD45RA $\left.{ }^{+} / \mathrm{HLA}_{-} \mathrm{DR}^{\text {neg }}\right)$, Activated (CD45RA $\left.{ }^{\text {neg }} \mathrm{HLA}^{-D R^{\text {neg }}}\right)$ and Effector $\left(\mathrm{CD} 45 \mathrm{RA}{ }^{\text {neg HLA- }}\right.$ $\left.\mathrm{DR}^{\text {low/high }}\right)$ Treg cells, being HLA-DR ${ }^{\text {high }}$ representative of highly suppressive terminally differentiated Tregs [23-31]. The last subtype of Treg (CD45RA $\left.{ }^{\text {neg } H L A-D R}{ }^{\text {high }}\right)$ loses its regulatory properties and produces inflammatory cytokines when CD25 expression is low/neg [16,23,24].

Hence, the immunophenotyping of functionally different Treg subsets can be assessed on non-permeabilized cells using the panel of six monoclonal antibodies specifically binding the six surface antigens CD4, CD25, CD127, CD45RA, CD39, and HLA-DR; the two latter providing a clue of the strength of the inhibitory function of Treg ex vivo, without need for in vitro assays [23,25-28,32,33] (Fig 1).

In the present study, the described analytical approach was applied to study Treg profile in mite allergic pediatric patients undergoing SLIT, in order verify a possible correlation between specific subsets of Treg and the effectiveness of SLIT. The AIT was done by monomeric allergoid, an acid-resistant immunotherapeutic tool known to elicit early T reg-associated cytokine modulation [3].

\section{PATIENTS - DIAGNOSIS AND TREATMENT}

The present is an observational prospective study approved by the local Ethical Committee of University "G. d'Annunzio", Chieti-Pescara (Protocol No. 22/2019), carried out according to the Declaration of Helsinki and subsequent revisions. All patients and parents signed a written informed consent after having been informed about the procedures of the study. 
2.1 Study design . 20 patients diagnosed with respiratory allergy (persistent rhinitis with or without asthma) to mites (positive skin test and specific IgE) were enrolled in the study. Patients already on SLIT, with severe allergic asthma or with co-sensitization to pollens were excluded.

At the enrollment, relevant anthropometric and laboratory data were gathered, allergic rhinitis (AR) was graded according to ARIA guidelines in 1) intermittent mild, 2) intermittent moderate/severe, 3) persistent mild and 4) persistent moderate/severe depending on the severity of symptoms and quality of life outcomes

[34] and concomitant asthma was graded by ACT quality of life questionnaire and spirometry eventually completed with bronchodilation test (BDT). At the enrollment, each patient marked in a $100 \mathrm{~mm}$ visual analogic scale (VAS) the level of its health status related to allergy with 0 the best status and 100 the worst. The outline of the study is shown in Table 1.

2.2 Treatment. All patients were treated by SLIT with mite monomeric allergoid (LAIS - Lofarma, Milan, Italy) at $1000 \mathrm{UA}$ four times per week every other day, for 12-months, that was preceded by 1-week induction phase. For safety assessment, any adverse local and systemic reactions were annotated with description of severity and frequency. The effectiveness of SLIT was established comparing VAS, ARIA grading and ACT questionnaire performed after 12-months of treatment with their basal values.

2.3 Blood sampling. Two blood samples were drawn at baseline (T0) and after 12-months of SLIT, processed within four hours and cryopreserved to be further analyzed for Regulatory T-cells by flow cytometry.

\section{METHODS}

\subsection{ISOLATION AND STORAGE OF PERIPHERAL BLOOD MONONUCLEAR CELLS}

From each enrolled subject, $12 \mathrm{ml}$ peripheral blood (PB) was drawn in EDTA $(2 \mathrm{mg} / \mathrm{mL}$ ) tubes (BD Vacutainer K2E, Becton Dickinson Biosciences, San Jose, CA, USA), and processed within 3 hours from bleeding. Density gradient centrifugation whole blood (diluted 1:1 with PBS) was carried out on a Lymphoprep cushion (Sentinel) in order to isolate peripheral blood mononuclear cells (PBMCs). The lympho-monocytes-enriched phase was gathered and washed; finally, single-cell suspensions were prepared using $1 \mathrm{ml}$ of cryogenic medium (90\% heat-inactivated FCS-10\% DMSO) and viably frozen at $-80^{\circ} \mathrm{C}$ for flow cytometry analysis of Treg cells [35].

The day before the cytofluorimetric analysis, viably cryopreserved samples (approximately 10x106 PBMC) were thawed, washed in PBS, and cultured overnight in RPMI 1640 medium (HyClone) supplemented with $10 \%$ heat-inactivated FCS (Hyclone), $10 \mathrm{mM}$ HEPES, $1 \%$ nonessential amino acids, $1 \mathrm{mM}$ sodium pyruvate, $100 \mathrm{U} / \mathrm{ml}$ penicillin, $100 \mathrm{ug} / \mathrm{ml}$ streptomycin, $2 \mathrm{mM}$ L-glutamine (Life Technologies, Gaithersburg, MD), in a humified incubator at $37^{\circ} \mathrm{C}, 5 \% \mathrm{CO}_{2}$.

\subsection{REGULATORY T-CELLS STAINING FOR FLOW CYTOMETRY IMMUNOPHENOTYPING}

Thawed and recovered PBMCs were processed by a common flow cytometry lyse and wash method [36]. Briefly, samples were stained using a panel of lyophilized reagents, as detailed in Table 2. After 30 min of staining $\left(4^{\circ} \mathrm{C}\right.$ in the dark), samples underwent an erythrocyte-lyse step, with $1 \mathrm{~mL}$ of $1 \mathrm{X}$ Pharm Lysing solution (BD, Biosciences), for 15 min at room temperature, under gentle agitation, according to manufacturer's instructions. Samples were then centrifuged $(400 \mathrm{~g}, 10 \mathrm{~min}$, room temperature) and washed by adding $2 \mathrm{ml}$ of $1 \mathrm{X}$ PBS and $1.5 \times 10^{5}$ events/sample were acquired by flow cytometry (FACSCanto, three laser, eight color configurations, BD Biosciences). The threshold was placed on the Forward Scatter (FSC) channel. Treg cells and their subsets were identified as described by other investigators [20,25]. Cytofluorimetric data were analyzed by using FACDiva v6.1.3 software (BD Biosciences). To ensure correct identification of negative and positive populations, cells were plotted using dot-plot bi-exponential display [36,37].

Instrument performances, data reproducibility and fluorescence calibrations were sustained and checked by the Cytometer Setup \& Tracking Module and further validated by the acquisition of Spherotech 8 peack Rainbow Beads (BD). To evaluate non-specific fluorescence, Fluorescence Minus One (FMO) controls were used $[38,39]$. Compensation was assessed using CompBeads (BD) and single stained fluorescent samples. Data 
were analyzed using FACSDiva v 6.1.3 (BD), FACSuite v 1.0.5 (BD) and FlowJo v 8.8.6 (TreeStar, Ashland, OR, USA) software. CD39 and HLA-DR surface expression, evaluated in terms of Mean Fluorescence Intensity (MFI), were normalized (MFI Ratio) based on the relative expression of the respective CD4 ${ }^{\text {neg }}$ lymphocyte compartment. In details, for Treg identification and subtyping, CD4 ${ }^{+} \mathrm{T}$ lymphocytes were firstly gated; CD4 ${ }^{+}$ T-cells were then analyzed for surface expression of CD25; then, based on detection of CD127, the CD25 high cell subset was further distinguished into Treg cells (CD127 $\left.{ }^{\text {neg }}\right)[25]$ and activated T-cells (CD127 high $)[26]$. Hence, the Treg subpopulation of $\mathrm{CD} 4{ }^{+} \mathrm{CD} 25^{+} \mathrm{CD} 39^{+} \mathrm{CD} 127^{\text {neg }}$ cells was analyzed as Resting Treg (rTreg) when CD45RA ${ }^{\text {pos }} /$ HLA-DR $^{\text {neg }}$, Activated Treg (aTreg) when CD45RA`HLA-DR ${ }^{\text {neg/low }}$, and Effector Treg (eTreg) when CD45RA ${ }^{\text {neg HLA-DR }}{ }^{\text {high }}$ (Table 3). The panel of antibodies used for Treg identification and subtyping was purchased as lyophilized pre-cast mixture (Lyotube \#624637, BD Biosciences).

Example of the gating strategy and representative distribution of the three subpopulations of Tregs based on the expression level of the extracellular CD markers CD4, CD25, CD127, CD45RA, HLA-DR and CD39 is shown in Figure 2.

\subsection{STATISTICAL ANALYSIS}

Data are expressed as median \pm interquartile range, unless otherwise indicated. All of the measured parameters, both clinical and immunological, had nonparametric distribution (according to Shapiro-Wilk's criteria), so statistical comparisons within each group separately at the time of the first SLIT administration (T0) and after 12 months of treatment (T12) were performed using non-parametric Wilcoxon signed-rank test. To assess the correlations between changes of each clinical parameter and relative abundance of each Treg cells subset, the Spearman test was used. $\mathrm{P}$ values $<0.05$ were considered statistically significant. Statistical analysis was performed using Prism 5.0 software.

4 Results

The demographic, clinical and laboratory parameters of patients are shown in Table 4 and Table 5.

\subsection{CHANGES IN CLINICAL PARAMETERS}

Clinical parameters significantly improved after SLIT: VAS and ARIA rhinitis score significantly decreased from $7.8 \pm 1.5$ to $4.3 \pm 1.8$ and from $3.70 \pm 0.5$ to $1.85 \pm 0.9$ respectively after SLIT, with the same statistical significance (Wilcoxon $\mathrm{z}-3.7236 ; \mathrm{p}=0.0002$ ). Improvement was evidenced also in the subgroup of asthmatic patients $(n=7)$, since ACT scores significantly increased from the baseline value of 18 (16-19) up to 24 (20-25) after 12 months of treatment $(\mathrm{p}<0.05)$. However, the number of patients is not sufficiently large to consider these data as demonstrative of the effectiveness of AIT; they are only descriptive of the clinical condition of patients after 12 months of treatment, to be compared with Treg analyses.

\subsection{TREGS SUBSETS ARE RE-PATTERNED AFTER EFFECTIVE 12-MONTHS ALLERGOID SLIT}

Tregs were analyzed as frequency of total Treg cells and their three subsets, namely Resting (rTregs), Activated (aTregs) and Effector (eTregs), within the parental population of $\mathrm{CD}^{+}$cells. Total Tregs did not change significantly; rTreg significantly decreased from $61.1 \% \pm 9$ to $59.1 \% \pm 10$ of the parental population (Wilcoxon z-3.6214, $\mathrm{p}<0.0003$ ), while, the abundance of aTregs significantly incremented from $5.8 \% \pm 4.4$ to $8.8 \% \pm 4.7$ and eTregs from $31.4 \% \pm 10.3$ to $37.6 \% \pm 8.9$ of the parental population (Wilcoxon $\mathrm{z}-2.9011, \mathrm{p}<0.05$ and $\mathrm{z}-3.077, \mathrm{p}=0.002$, respectively) (Fig $3 \mathrm{~A}$ and Fig $3 \mathrm{~B}$ ). A significant negative correlation has been observed between the decrease in rTreg and the increase in aTreg (Spearman's $p=-0.69391, p<0.02$.) and increase in eTreg cells (Spearman's $\rho=-0.56845, \mathrm{p}<0.02$ ) (Fig 4A and Fig 4B).

\subsection{HLA-DR AND CD39 LEVELS ARE UP-REGULATED IN ACTIVATED AND EFFECTOR TREGS} AFTER SLIT

HLA-DR, Treg maturation and activation marker, was analyzed as level of expression on total Tregs, and in particular in each Treg subset, at both timepoints of the study, before and after 12 months SLIT. HLA-DR resulted significantly up-regulated in all Tregs (Fig 5) from 4.93 \pm 3.1 to $6.92 \pm 5.1$ MFI (Wilcoxon z-4.2026, p 
$<0.00001$ ). In particular, HLA-DR increased on aTregs from $3.4 \pm 3.03$ to $4.91 \pm 3.2$ MFI (Wilcoxon z-3.2479, $\mathrm{p}=0.001$ ) and on eTregs from $1.54 \pm 0.66$ to $2.0 \pm 1.45 \mathrm{MFI}$ (Wilcoxon $\mathrm{z}-2.9664, \mathrm{p}=0.005$.) (Fig 6).

CD39 expression, functional marker of Treg suppressive/anti-inflammatory activity, was assessed at the surface of total Tregs and their subsets, comparing its expression in the cohort of AR patients at the time of enrolment (baseline, T0) and after 12 months of treatment (T12). As expected, at baseline, CD39 was found differently expressed in the three subsets of Tregs with Resting < Activated $<$ Effector. After 12 months of SLIT, CD39 surface expression was found significantly increased in all Tregs (Fig 5) from $6.9 \pm 4$ to $8.02 \pm 5$ MFI (Wilcoxon z-3.1049, p=0.001) (Fig 5).

\subsection{CORRELATION OF CLINICAL SCORES AND REGULATORY T-CELLS SUBSETS}

Changes in HLA-DR expression on all Treg cells significantly correlated with variation in VAS pre-/postSLIT (Spearman's $p=0.54104, p(2$-tailed $)=0.01376)($ Fig 7$)$. Changes in eTregs was already significantly correlated with both ARIA (Spearman's $\rho=0.58728, p=0.013$ ) (Fig 8A) and VAS (Spearman's $\rho=0.49172$, $\mathrm{p}=0.044$.) (Fig $8 \mathrm{~B}$ ) variations after SLIT. While a significant negative correlation was found between $\mathrm{rTregs}$ and clinical parameter changes after treatment (Spearman's $p=-0.48482, p=0.0491$ ).

No other correlations were found except that the lowest increase (less than 8\%) of memory Treg (CD45RA ${ }^{\text {neg }}$ cells) appears to characterize patients with the lowest specific mite IgE serum levels.

\section{Discussion}

According to the current knowledge, AIT leads to immune tolerance through the induction of allergenspecific Treg, FoxP3 $3^{+} / \mathrm{CD} 127^{\text {low/neg }}$ T-cells, able to suppress ILC2, Th2 cells and IgE-expressing B-cells through cell-cell contact and/or inhibitory cytokine production [40]. Our results suggest that, during SLIT with monomeric allergoids, allergen specific Tregs are CD $4^{+} \mathrm{CD} 25^{\text {high }} \mathrm{CD} 39^{+} \mathrm{CD} 127^{\text {neg }} \mathrm{HLA}-\mathrm{DR}{ }^{\mathrm{pos}}$ and CD45RA ${ }^{\text {neg. }}$. In particular, the differentiation of Resting Tregs into Activated/Effector memory Treg cells directly correlates with effective SLIT with monomeric allergoids. To our knowledge, this is first report on successful SLIT being associated with re-patterning of the differentiation status of Tregs.

It is well established that prevention of airways allergic diseases occurs early in life and relies on the acquisition of a specialized subtype of $\mathrm{CD} 4^{+} \mathrm{T}$-cells with regulatory functions and allergen specificity. Some authors speculate that, in early life, a relatively immature immune system could permit the development of inducible Tregs specific to a spectrum of allergens, contrasting allergy [41]. But, when an allergic response is already set by Th2 effector cells, de novo generation of Tregs is hampered by Th2 allergic cytokines, IL-4 and IL-6, that inhibit the induction of Foxp3, the specific transcription factor that make naive $\mathrm{CD}^{+}$cells become Treg. Enhancement of Tregs has been described in seasonal rhinitis [42], but these cells are underrepresented in the blood of AR children [43]. Recently, AR children have been shown that Foxp3 ${ }^{+}$Tregs have defect in suppressing IgE production (in vitro) and that they can be incremented by mite SLIT; notably, in these patients follicular and peripheral Foxp3 ${ }^{+}$Tregs are phenotypically identical [15].

Since their discovery, the study of Tregs role in immune mechanisms is based on the detection of the specific Foxp3 transcription factor; however, in inflammatory conditions, FoxP $3^{+}$Treg might become unstable and halt the production of their functional suppressive cytokines [44]. Phenotypically indistinguishable $\mathrm{CD} 4{ }^{+} \mathrm{CD} 25^{\text {high }} \mathrm{FoxP} 3^{+} \mathrm{T}$-cells, somewhat functionally redundant, have been described differentiating from naïve $\mathrm{CD} 4^{+} \mathrm{T}$-cells in the thymus, in response to self-antigens, or in the periphery in response to foreign antigens [45]; delayed maturation of Tregs is suspected to predispose atopic children [46] and non-atopic individuals [41] to allergic diseases. The most prevalent subset of human $\mathrm{CD} 4^{+}$Tregs, the natural Tregs, is characterized by undetectable or low level of CD127 antigen on the cell surface, that inversely correlates with specific intracellular marker FoxP3 and the suppressive function [16,25,26]. Interestingly, in newborns, thymic CD127 $7^{\text {neg }}$ Tregs can suppress the proliferative response to allogenic stimulation of $\mathrm{CD} 4^{+}\left(\mathrm{CD} 25^{\text {neg }}\right)$ T-cells in a dose-dependent manner [18].

Therefore, in the present study, Tregs were identified as CD127 ${ }^{\text {neg }}$ cells within the CD $4^{+} \mathrm{CD} 25^{\text {high }}$ T-cells 
compartment to surmount unpredictable FoxP3 stability and, meanwhile, to avoid potential technical artifacts associated with intracellular Foxp3 detection.

To better characterize the CD127 ${ }^{\text {neg }} \mathrm{T}$ reg subsets, the CD45RA determinant as well as HLA-DR and CD39 expression were analyzed. Of interest for our study are CD45RA ${ }^{\text {neg }}$ Tregs. They are memory cells enabled to survive for long periods of time, even in absence of the specific antigen, showing increased activity upon reexposure to it (recall immune response) and able to induce apoptosis in target cells [47]. HLA-DR expression on Tregs can be negative, low, or high. Precisely, HLA-DR ${ }^{\text {neg }}$ are Resting Tregs: in the periphery, they are likely yet to encounter their cognate antigen or constantly being exposed to antigen below the activation threshold; by contrast, HLA-DR ${ }^{\text {low/high }}$ Tregs are Activated/Effector cells upon strong antigen stimulation outside the thymus, also involved in the homeostatic maintenance of Tregs in vivo [48].

HLA-DR expression identifies a functionally distinct population of what appear to be terminally differentiated human Tregs. FoxP3 expression is significantly higher in the HLA-DR ${ }^{+}$Tregs [23]. These cells exhibit earlier kinetics of responder T-cells (Tresp) suppression than the HLA-DR ${ }^{\text {neg }}$ subset. This suppression is contactdependent, and these cells do not produce IL-10. The action of these cells is unique from that of HLA-DRTregs, which induce a later, less vigorous suppression of Tresp, and which rely on both cell-contact and IL-10 secretion to inhibit Tresp activation [23].

HLA-DR expression also determines the extent and the kinetic of the suppressive function: for example, in renal transplant patients, the level of HLA-DR expression of CD45RA ${ }^{\text {neg }}$ Treg cell subset correlated positively with the suppressive activity of the total Treg cell pool, high levels of circulating HLA-DR ${ }^{\text {pos }}$ Treg cells represent an in vitro predictive biomarker of allograft tolerance, while Foxp $3^{+}$detection alone lacks specificity and sensitivity [18]; in mice, the HLA-DR ${ }^{\text {neg }}$ Treg initially skew the immune response toward the production of Th2 cytokines, before initiating late suppression; whereas, the HLA-DR ${ }^{\text {pos }}$ Treg population exhibits early suppression $[23,49]$. Moreover, it has been demonstrated that HLA-DR ${ }^{\text {pos }}$ Treg cells tend to present higher gene expression of molecules associated with contact-dependent cell activation and cytotoxicity in patients with allergy [29].

In summary, Tregs can be subtyped as Resting $\left(\mathrm{CD} 4{ }^{+} \mathrm{CD} 25^{+} \mathrm{CD} 39^{+} \mathrm{CD} 127^{\text {neg }} \mathrm{CD} 45 \mathrm{RA}{ }^{\text {pos }} / \mathrm{HLA}\right.$ $\left.\mathrm{DR}^{\text {neg }}\right)$, Activated $\left(\mathrm{CD} 4^{+} \mathrm{CD} 25^{+} \mathrm{CD} 39^{+} \mathrm{CD} 127^{\text {neg }} \mathrm{CD} 45 \mathrm{RA}^{\text {neg }} \mathrm{HLA}-\mathrm{DR}{ }^{\text {neg }} / \mathrm{low}\right), \quad$ and Effector $\left(\mathrm{CD} 4{ }^{+} \mathrm{CD} 25^{+} \mathrm{CD} 39^{+} \mathrm{CD} 127^{\text {neg }} \mathrm{CD} 45 \mathrm{RA}^{\text {neg }} \mathrm{HLA}-\mathrm{DR}{ }^{\text {high }}\right)$. This last subtype can be considered effector Treg cells part of the activated Treg compartment of which they seem to constitute a terminally differentiated subset, with a more efficient and rapid suppressive function compared to other subtypes [23,31]. They are different from secreting or type III Tregs that some authors define as non-suppressive cells and represent a short-lived terminally differentiated population, which is divided rapidly and disappears [16,23,31]. Suppressive Tregs are CD127 $7^{\text {neg }}$ while type III Tregs are CD127 low/high. As described, CD127 expression inversely correlates with FoxP3 expression and the suppressive function in Tregs.

CD39 has been recently described as a Treg functional marker. It is an ectonucleotidase producing the inhibitory and antiproliferative AMP, by hydrolysing the pro-inflammatory ATP released by damaged cells [22] that contributes to the suppressive activity of the Treg of the memory subset [50], is upregulated upon TCR ligation, [51] and is tunable on Tregs (studied in $\mathrm{HIV}^{+}$subjects) [28].

CONCLUDING REMARKS. In line with these concepts, in our study we observed a reduction of Resting Treg and higher rates of Activated/Effector Tregs after SLIT. Remarkedly, higher levels of the surface HLADR determinant and of the CD39 markers, both playing inhibitory function in Tregs, were detected at higher level on Tregs after effective mite-allergoid SLIT, suggesting that SLIT also induced empowerment of Treg inhibitory capacity, likely compensating the under-representation of Treg observed in allergic patients [43].

Our study was not designed to evaluate the effectiveness of the mite-allergoid SLIT, already demonstrated by other authors by specific devoted trials [52]; rather, our goal was to associate the variations of Tregs subsets with successful SLIT. In our study, variable clinical outcomes were observed in patients, from complete to partial or no remission of symptoms. Notably, the increase of HLA-DR expression on Tregs, Activated/Effector Tregs in particular, positively correlates with the obtained clinical improvement evaluated by both patients 
(VAS) and clinicians (ARIA score). The significant negative correlation $(\mathrm{p}=0.049)$ between Resting Tregs and the above-mentioned clinical parameters confirms these data.

The immunophenotypic assessment of Treg subsets described in the present study, formerly applied to shed light into their immunological mechanism of different pathological and therapeutic conditions, also provides a new insight of the immune-modulating effects of mite-allergoid SLIT and might also represent an analytical tool for the monitoring of SLIT efficacy. The next step of our study will be the detection of changes in Tregs after the first months of AIT to be used as markers for the early identification of responders/non-responders by mean of a straightforward and not invasive blood test.

\section{CONFLICTS OF INTEREST}

GM and EC are employed by Lofarma. All the other authors declare no conflicts of interest.

\section{AUTHORS CONTRIBUTION}

CP, GM and MDG conceived and designed the study, pursued the Ethical Committee approval process, analyzed the data, and contributed to the article writing.

SDP and MP performed the clinical procedures, blood samples collections and accomplished ethical issues.

CP, PL, MM and LP designed the antibody panel and the FACS analysis setup, performed storage, processing, staining of the blood cells and performed the serological determinations.

RP, TO, MM, PL and EC analyzed the data and contributed to the writing.

ORCID

Claudia Petrarca ORCID:https://orcid.org/0000-0002-6910-0160

\section{REFERENCES}

Tosca MA, Licari A, Olcese R, Marseglia G, Sacco O, Ciprandi G. Immunotherapy and Asthma in Children. Front Pediatr. 2018;6:231. doi:10.3389/fped.2018.00231.

1. Canonica GW, Cox L, Pawankar R, Baena-Cagnani CE, Blaiss M, Bonini S et al. Sublingual immunotherapy: World Allergy Organization position paper 2013 update. World Allergy Organ J. 2014;7(1):6. doi:10.1186/1939-4551-7-6.

2. Di Gioacchino M, Cavallucci E, Ballone E, Cervone M, Di Rocco P, Piunti E et al. Dose-dependent clinical and immunological efficacy of sublingual immunotherapy with mite monomeric allergoid. Int J Immunopathol Pharmacol. 2012;25(3):671-9. doi: 10.1177/039463201202500313.

3. Petrarca C, Lazzarin F, Lanuti P, Marchisio M, Miscia S, Rossi C et al. Lactobacillus paracasei Lp6 favors immune modulation induced by allergoid treatment in ragweed sensitized mice. Int J Immunopathol Pharmacol. 2011;24(4):881-93. doi:10.1177/039463201102400407.

4. Petrarca C, Clemente E, Amato V, Gatta A, Cortese S, Lamolinara A et al. Vitamin D3 improves the effects of low dose Der p 2 allergoid treatment in Der p 2 sensitized BALB/c mice. Clin Mol Allergy. 2016;14(1):7. doi:10.1186/s12948-016-0044-1.

5. Di Gioacchino M, Perrone A, Petrarca C, Di Claudio F, Mistrello G, Falagiani P et al. Early cytokine modulation after the rapid induction phase of sublingual immunotherapy with mite monomeric allergoids. Int J Immunopathol Pharmacol. 2008;21(4):969-76. doi:10.1177/039463200802100421.

6. Passalacqua G, Albano M, Fregonese L, Riccio A, Pronzato C, Mela GS et al. Randomised controlled trial of local allergoid immunotherapy on allergic inflammation in mite-induced rhinoconjunctivitis. Lancet. 1998;351(9103):629-32. doi:10.1016/S0140-6736(97)07055-4.

7. Akdis CA, Akdis M. Mechanisms of allergen-specific immunotherapy and immune tolerance to allergens. World Allergy Organ J. 2015;8(1):1-12. doi:10.1186/s40413-015-0063-2.

8. Winkler B, Hufnagl K, Spittler A, Ploder M, Kallay E, Vrtala S et al. The role of Foxp3+ T cells in long-term efficacy of prophylactic and therapeutic mucosal tolerance induction in mice. Allergy. 2006;61(2):173-80. doi:10.1111/j.1398-9995.2006.01014.x. 
9. Gueguen C, Bouley J, Moussu H, Luce S, Duchateau M, Chamot-Rooke J et al. Changes in markers associated with dendritic cells driving the differentiation of either TH2 cells or regulatory $\mathrm{T}$ cells correlate with clinical benefit during allergen immunotherapy. J Allergy Clin Immunol. 2016;137(2)545558. doi:10.1016/j.jaci.2015.09.015.

10. Petrarca C, Lazzarin F, Pannellini T, Iezzi M, Braga M, Mistrello G et al. Monomeric allergoid intragastric administration induces local and systemic tolerogenic response involving IL-10-producing CD4(+)CD25(+) Regulatory T cells in mice. Int J Immunopathol Pharmacol. 2010;23(4):1021-31. doi:10.1177/039463201002300407.

11. Petrarca C, Clemente E, Toto V, Iezzi M, Rossi C, Zanotta S et al. rBet v 1 immunotherapy of sensitized mice with Streptococcus thermophilus as vehicle and adjuvant. Hum Vacc Immunother. 2014;10(5):1228-37. doi:10.4161/hv.28155.

12. Zimmer A, Bouley J, Le Mignon M, Pliquet E, Horiot S, Turfkruyer M et al. A regulatory dendritic cell signature correlates with the clinical efficacy of allergen-specific sublingual immunotherapy. J Allergy Clin Immunol. 2012;129:1020-1030. doi:10.1016/j.jaci.2012.02.014.

13. Suarez-Fueyo A, Ramos T, Galan A, Jimeno L, Wurtzen PA, Marin A et al. Grass tablet sublingual immunotherapy downregulates the TH2 cytokine response followed by regulatory T-cell generation. J Allergy Clin Immunol. 2014;33(1):130-138.e2. doi:10.1016/j.jaci.2013.09.043.

14. Yao Y, Wang ZC, Wang N, Zhou PC, Chen CL, Song J et al. Allergen immunotherapy improves defective follicular regulatory $\mathrm{T}$ cells in patients with allergic rhinitis. J Allergy Clin Immunol. 2019;144(1):118-128. doi:10.1016/j.jaci.2019.02.008.

15. Miyara M, Yoshioka Y, Kitoh A, Shima T, Wing K, Niwa A et al. Functional delineation and differentiation dynamics of human $\mathrm{CD} 4+\mathrm{T}$ cells expressing the FoxP3 transcription factor. Immunity. 2009;30(6):899-911. doi:10.1016/j.immuni.2009.03.019.

16. Biancotto A, Dagur PK, Fuchs JC, Wiestner A, Bagwell CB, McCoy JP Jr. Phenotypic complexity of Regulatory T subsets in patients with B-chronic lymphocytic leukemia. Mod Pathol. 2012;25(2):24659. doi:10.1038/modpathol.2011.164.

17. Gronert Alvarez A, Fytili P, Suneetha PV, Kraft AR, Brauner C, Schlue J et al. Comprehensive phenotyping of regulatory $\mathrm{T}$ cells after liver transplantation. Liver Transplant. 2015;21(3):381-95. doi:10.1002/lt.24050.

18. Chiappori A, Folli C, Balbi F, Caci E, Riccio AM, De Ferrari L et al. CD4+CD25highCD127-regulatory T-cells in COPD: smoke and drugs effect. World Allergy Organ J. 2016;9(1):5. doi:10.1186/s40413016-0095-2.

19. Khosravi M, Majdinasab N, Amari A, Ghadiri AA. Increased frequency of CD4+CD25high CD127low/ - regulatory T cells in patients with multiple sclerosis. Gene Rep. 2019;17:100456. doi:10.1016/j.genrep.2019.100456.

20. Oberstein TJ, Taha L, Spitzer P, Hellstern J, Herrmann M, Kornhuber J et al. Imbalance of Circulating Th17 and Regulatory T Cells in Alzheimer's Disease: A Case Control Study. Front Immunol. 2018;9:1213. doi:10.3389/fimmu.2018.01213.

21. Deaglio S, Dwyer KM, Gao W, Friedman D, Usheva A, Erat A et al. Adenosine generation catalyzed by CD39 and CD73 expressed on regulatory T cells mediates immune suppression. J Exp Med. 2007;204(6):1257-1265. doi:10.1084/jem.20062512.

22. Baecher-Allan C, Wolf $\mathrm{E}$ and Hafler DA. MHC class II expression identifies functionally distinct human regulatory T cells. J Immunol. 2006:176(8):4622-31. doi:10.4049/jimmunol.176.8.4622.

23. Cuadrado E, van den Biggelaar M, de Kivit S, et al. Proteomic Analyses of Human Regulatory T Cells Reveal Adaptations in Signaling Pathways that Protect Cellular Identity. Immunity. 2018;48:104659.e6. doi:10.1016/j.immuni.2018.04.008

24. Liu W, Putnam AL, Xu-Yu Z, Szot GL, Lee MR, Zhu S et al. CD127 expression inversely correlates with FoxP3 and suppressive function of human CD4+ T reg cells. J Exp Med. 2006;203(7):1701-11. doi:10.1084/jem.20060772.

25. Seddiki N, Santner-Nanan B, Martinson J, Zaunders J, Sasson S, Landay A et al. Expression of interleukin (IL)-2 and IL-7 receptors discriminates between human regulatory and activated T cells. J 
Exp Med. 2006;203(7):1693-700. doi:10.1084/jem.20060468.

26. Orru V, Steri M, Sole G, Sidore C, Virdis F, Dei M et al. Genetic variants regulating immune cell levels in health and disease. Cell. 2013;155(1):242-56. doi:10.1016/j.cell.2013.08.041.

27. Simonetta F, Bourgeois C. CD4+FOXP3+ Regulatory T-Cell Subsets in Human Immunodeficiency Virus Infection. Front Immunol. 2013;4:215. doi: 10.3389/fimmu.2013.00215.

28. Geraldes L, Morgado J, Almeida A, Todo-Bom A, Santos P, Paiva A, Cheira C, Pais ML. Expression patterns of HLA-DR + or HLA-DR- on CD4+/CD25++/CD127low regulatory T cells in patients with allergy. J Investig Allergol Clin Immunol 2010;20(3):201-9.

29. Kotsakis A, Koinis F, Katsarou A, et al. Prognostic value of circulating regulatory T cell subsets in untreated non-small cell lung cancer patients. Sci Rep. 2016;6:39247. Published 2016 Dec 15. doi:10.1038/srep39247

30. Schaier M, Seissler N, Schmitt E, et al. DR(high+)CD45RA(-)-Tregs potentially affect the suppressive activity of the total Treg pool in renal transplant patients. PLoS One. 2012;7(3):e34208. doi:10.1371/journal.pone.0034208

31. Deaglio S, Robson SC. Ectonucleotidases as regulators of purinergic signaling in thrombosis, inflammation, and immunity. Adv Pharmacol. 2011;61:301-32. doi:10.1016/B978-0-12-385526-8.00010-2.

32. Zhao ST, Wang CZ. Regulatory T cells and asthma. J Zhejiang Univ Sci B. 2018;19(9):663-673. doi:10.1631/jzus.B1700346.

33. Brożek JL, Bousquet J, Agache I, Agarwal A, Bachert C, Bosnic-Anticevich S et al. Allergic Rhinitis and its Impact on Asthma (ARIA) guidelines - 2016 revision. J Allergy Clin Immunol. 2017;140(4):950-958. doi:10.1016/j.jaci.2017.03.050.

34. Nettenstrom L, Alderson K, Raschke EE, Evans MD, Sondel PM, Olek S et al. An optimized multiparameter flow cytometry protocol for human Regulatory $\mathrm{T}$ cell analysis on fresh and viably frozen cells, correlation with epigenetic analysis, and comparison of cord and adult blood. J Immunol Methods. 2013;387(1-2):81-8. doi:10.1016/j.jim.2012.09.014.

35. Lanuti P, Ciccocioppo F, Bonanni L, Marchisio M, Lachmann R, Tabet N et al. Amyloid-specific T-cells differentiate Alzheimer's disease from Lewy body dementia. Neurobiol Aging. 2012;33(11):2599-611. doi:10.1016/j.neurobiolaging.2012.01.004.

36. Lanuti P, Rotta G, Almici C, Avvisati G, Budillon A, Doretto P et al. Endothelial progenitor cells, defined by the simultaneous surface expression of VEGFR2 and CD133, are not detectable in healthy peripheral and cord blood. Cytom Part A- 2016;89(3). doi:10.1002/cyto.a.22730.

37. Lachmann R, Lanuti P and Miscia S. OMIP-011: Characterization of circulating endothelial cells (CECs) in peripheral blood. Cytom Part A. 2012;81(7):549-51. doi:10.1002/cyto.a.22071.

38. Lanuti P, Santilli F, Marchisio M, Pierdomenico L, Vitacolonna E, Santavenere E et al. A novel flow cytometric approach to distinguish circulating endothelial cells from endothelial microparticles: relevance for the evaluation of endothelial dysfunction. Journal Immunol Methods. 2012;380(1-2):1622. doi:10.1016/j.jim.2012.03.007.

39. Shamji MH, Durham SR. Mechanisms of allergen immunotherapy for inhaled allergens and predictive biomarkers. J Allergy Clin Immunol. 2017;140(6):1485-1498. doi:10.1016/j.jaci.2017.10.010.

40. Zhang H, Kong H, Zeng X, Guo L, Sun X, He S. Subsets of regulatory T cells and their roles in allergy. J Transl Med. 2014;12(1):125. doi:10.1186/1479-5876-12-125.

41. Palmer C, Mulligan JK, Smith SE, Atkinson C. The role of regulatory T cells in the regulation of upper airway inflammation. Am J Rhinol Allergy. 2017;31(6):345-351. doi:10.2500/ajra.2017.31.4472.

42. Saad K, Zahran AM, Elsayh KI, Abdelmoghny A, Aboul-Khair MD. Variation of Regulatory T Lymphocytes in the Peripheral Blood of Children with Allergic Rhinitis. Arch Immunol Ther Exp. 2018;66(4):307-313. doi:10.1007/s00005-017-0498-y.

43. Sakaguchi S, Vignali DA, Rudensky AY, Niec RE, Waldmann H. The plasticity and stability of regulatory T cells. Nat Rev Immunol. 2013;13(6):461-467. doi:10.1038/nri3464.

44. Chatenoud L. Natural and Induced T CD4+CD25+FOXP3+ Regulatory T Cells. Methods Mol Biol. 2011;677:3-13. doi:10.1007/978-1-60761-869-0_1.

45. Stelmaszczyk-Emmel A, Zawadzka-Krajewska A, Szypowska A, Kulus M, Demkow U. Frequency and 
Activation of CD4+CD25 high FoxP3+ Regulatory T Cells in Peripheral Blood from Children with Atopic Allergy. Int Arch Allergy Immunol. 2013;162:16-24. doi:10.1159/000350769.

46. Rosenblum MD, Way SS and Abbas AK. Regulatory T cell memory. Nat Rev Immunol. 2016:16(2):90101. doi:10.1038/nri.2015.1.

47. Shevyrev D, Tereshchenko V. Treg Heterogeneity, Function, and Homeostasis. Front Immunol. 2020 Jan 14;10:3100. doi:10.3389/fimmu.2019.03100.

48. Ashley CW and Baecher-Allan C. Cutting Edge: Responder T Cells Regulate Human DR+ Effector Regulatory T Cell Activity via Granzyme B. J Immunol. 2009;183(8):4843-4847. doi:10.4049/jimmunol.0900845.

49. Mandapathil M, Lang S, Gorelik E, Whiteside TL. Isolation of functional human regulatory T cells (Treg) from the peripheral blood based on the CD39 expression. J Immunol Methods. 2009;346(12):55-63. doi:10.1016/j.jim.2009.05.004.

50. Borsellino G, Kleinewietfeld M, Di Mitri D, Sternjak A, Diamantini A, Giometto R et al. Expression of ectonucleotidase CD39 by Foxp3+ Treg cells: hydrolysis of extracellular ATP and immune suppression. Blood. 2007;110(4):1225-32. doi:10.1182/blood-2006-12-064527.

51. Pfaar O, Gerth van Wijk R, Klimek L, Bousquet J, Creticos PS. Clinical trials in allergen immunotherapy in the age group of children and adolescents: current concepts and future needs. Clin Transl Allergy. 2020;10:11. doi:10.1186/s13601-020-00314-1.

Table 1 . Outline of the study

\begin{tabular}{llll}
\hline & Time & Time & Time \\
\hline & T0 (enrolment) & T1 (day 0) & T3 (12 months) \\
Medical record & X & X & X \\
Prick test & X & & - \\
Serum total and & X & & - \\
specific IgE and blood & & & \\
tests & & & $\mathrm{X}$ \\
Visual Analogic Scale & $\mathrm{X}$ & & $\mathrm{X}$ \\
Rhinitis classification & $\mathrm{X}$ & & $\mathrm{X}$ \\
ARIA & $\mathrm{X}$ & & \\
Asthma control test - & & Start & ongoing \\
ACT & - & $\mathrm{X}$ & $\mathrm{X}$ \\
SLIT & - & & \\
Blood withdrawal for & & & \\
Tregs analysis & & & \\
\hline
\end{tabular}

Table 2. List of flow cytometry specificities and reagents.

\begin{tabular}{lll}
\hline Detection antigen & Antibody Clone & Fluorochrome \\
\hline CD4 & SK3 & APC-H7 \\
CD25 & 2A3 & PE \\
CD127 & HIL-7R-M21 & PerCP-Cy ${ }^{\text {TM }} 5.5$ \\
CD39 & TU66 & APC \\
CD45RA & L48 & FITC \\
HLA-DR & L243 & PE-Cy $^{\text {TM }} 7$ \\
\hline
\end{tabular}

Keys: Fluorescein Isothiocyanate (FITC); R-phycoerythrin; (PE); Peridinin-chlorophyll protein- Cyanine 5.5 (PerCP-Cy 5.5); Allophycocyanin (APC); APC-Hilite $\AA 7$ (APC-H7). All reagents were purchased by 
Becton Dickinson (BD) Biosciences (San Jose, CA, USA).

Table 3 . Panel of CD antigens targeted for the immunophenotypic detection and the assignment of the putative function of $\mathrm{T}$ reg cells and their subsets used in the present study $\left({ }^{2,3}\right)$.

\begin{tabular}{|c|c|c|c|c|c|c|}
\hline $\begin{array}{l}\text { Surface } \\
\text { Marker }\end{array}$ & CD4 & CD25 & CD127 & CD39 & CD45RA & HLA-DR \\
\hline $\begin{array}{l}\text { Conventional } \\
\text { CD4 T cells } \\
\text { Regulatory } \\
T \text { cells }\end{array}$ & + & - & high & - & + & - \\
\hline Resting & + & low $\$$ & low/neg\# & + & + & - \\
\hline Activated* & + & high & low/neg\# & + & - & - \\
\hline Effector* & + & high & low/neg\# & + & - & + \\
\hline
\end{tabular}

\# putative Foxp3 ${ }^{+}$cells

*Memory Regulatory T-cells

$\$$ CD25 ${ }^{\text {low }}$ Tregs can be either induced or naturally occurring

Table 4. Demographic data and IgE levels of patients at baseline $(n=20)$. The data are presented as median values together with their range (minimum-maximum) or as $\% \pm \mathrm{SD}$.

\begin{tabular}{ll}
\hline Gender, male/female & $16 / 4$ \\
\hline Age distribution, y & $11.50(6.5-17.40)$ \\
Rhinitis (concomitant asthma) & $20(7)$ \\
Total IgE $(\mathrm{kU} / \mathrm{l})(\mathrm{n}=12)$ & $196(33.3-1680)$ \\
sIgE to $D$. pteronyssinus $\mathrm{DP}(\mathrm{kU} / \mathrm{l})$ & $49.65(14.9->100)$ \\
sIgE to $D$. farinae $\mathrm{DF}(\mathrm{kU} / \mathrm{l})$ & $39.9(1.4->100)$ \\
\hline
\end{tabular}

Table 5. Respiratory clinical data and Treg frequency of patients pre- and post-SLIT ( $\mathrm{n}=20$ ). Tregs values represent the frequency of the parental CD4+ population. The data are presented as median values together with their range (minimum-maximum) or as $\% \pm \mathrm{SD}$.

\begin{tabular}{lll}
\hline & Pre-SLIT & Post-SLIT \\
\hline ARIA score & $3.7(2-4)$ & $1.85(0-3)$ \\
ACT score in asthmatics $(\mathrm{n} 7)$ & $18.2(16-19)$ & $23.14(20-25)$ \\
VAS & $7.8(3-10)$ & $4.3(1-7)$ \\
Resting Tregs & $61.1 \pm 9$ & $59.1 \pm 10$ \\
Activated Tregs & $5.8 \% \pm 4.4$ & $8.8 \% \pm 4.7$ \\
Effector Tregs & $31.4 \% \pm 10.3$ & $37.6 \% \pm 8.9$ \\
\hline
\end{tabular}

Figure legends

Figure 1.

Regulatory T-cells immunophenotyping strategy used in this study for subsets identification

Lymphomonocytes are selected on morphological characteristics parameters FSC/SSC. Then, a hierar- 
chical gating strategy based on six-color flow cytometric detection has been performed: the total Treg cell pool is identified by gating $\mathrm{CD} 4{ }^{+} \mathrm{CD} 25^{\text {high }} \mathrm{CD} 39^{+} \mathrm{CD} 127^{\text {low }} /{ }^{\text {neg }}$ cells; then, the gated-in events are further subtyped as Resting Treg (CD45RA $\left.{ }^{\text {pos }} \mathrm{DR}^{\text {neg }}\right)$, Activated Treg (CD45RA ${ }^{\text {neg }}{ }^{\text {DReg }}{ }^{\text {neg }}$ ) and Effector Treg $\left(\mathrm{CD} 45 \mathrm{RA}^{\text {neg }} \mathrm{DR}^{\text {pos }}\right)$. The names of the relevant markers of Tregs and their functional roles (i.e. effector, inhibitory, memory) are indicated.

Figure 2.

\section{Representative FACS analysis Tregs profile changes following SLIT}

Representative re-patterning of the three subpopulations of Tregs, Resting, Activated, and Effector, in one patient pre- and post-SLIT.

Figure 3.

\section{Changes in Treg subsets pre/post SLIT}

A) Relative abundance of total Tregs and of each subset expressed as median \pm interquartile range percent of the parent population. B) Delta-Treg frequency of each Treg subset pre/post 12 months SLIT. Statistical significance evaluated by non-parametric Wilcoxon signed-rank test.

Figure 4.

Correlations between Resting and Activated (A) and Effector (B) Tregs

Analysis of Treg subsets after SLIT shows a significant negative correlation between Resting and Memory Tregs (Activated and Effector). Statistical significance evaluated by Spearman's Rank Correlation.

Figure 5.

\section{Changes in HLA-DR and CD39 expression on Tregs pre/post SLIT}

Both HLA-DR and CD39 expression significantly increased in Tregs after 12 months SLIT.

Relative abundance of Tregs are expressed as median \pm interquartile range percent of the parent population. Statistical significance evaluated by non-parametric Wilcoxon signed-rank test.

Figure 6.

Changes in HLA-DR expression levels on Activated (A) and Effector (B) Tregs pre/post SLIT.

HLA-DR expression levels are significantly increased after SLIT both in Activated and in Effector Tregs. Relative abundance of Tregs are expressed as median \pm interquartile range percent of the parent population. Statistical significance evaluated by non-parametric Wilcoxon signed-rank test.

Figure 7.

Correlation between changes VAS and changes in HLA-DR expression on Tregs after SLIT.

Changes in HLA-DR expression on Regulatory T-cells after mite allergoid SLIT significantly correlates with changes in VAS. Statistical significance evaluated by Spearman's Rank Correlation.

Figure 8.

Correlation between clinical parameters and changes in Effector Regulatory T-cells.

Changes in Effector Regulatory T-cells after mite allergoid SLIT significantly correlates with changes in Aria classification for Rhinitis (A) and VAS (B). Statistical significance evaluated by Spearman's Rank Correlation. 


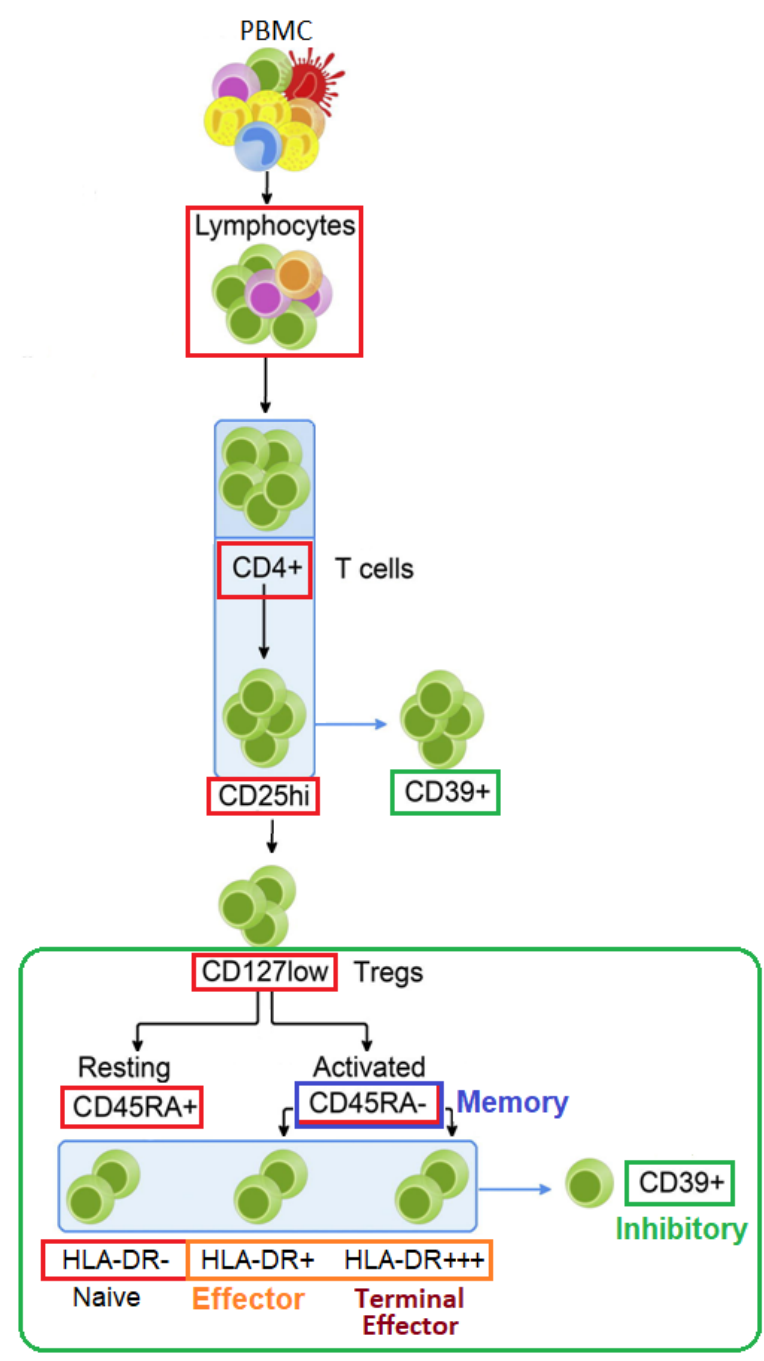



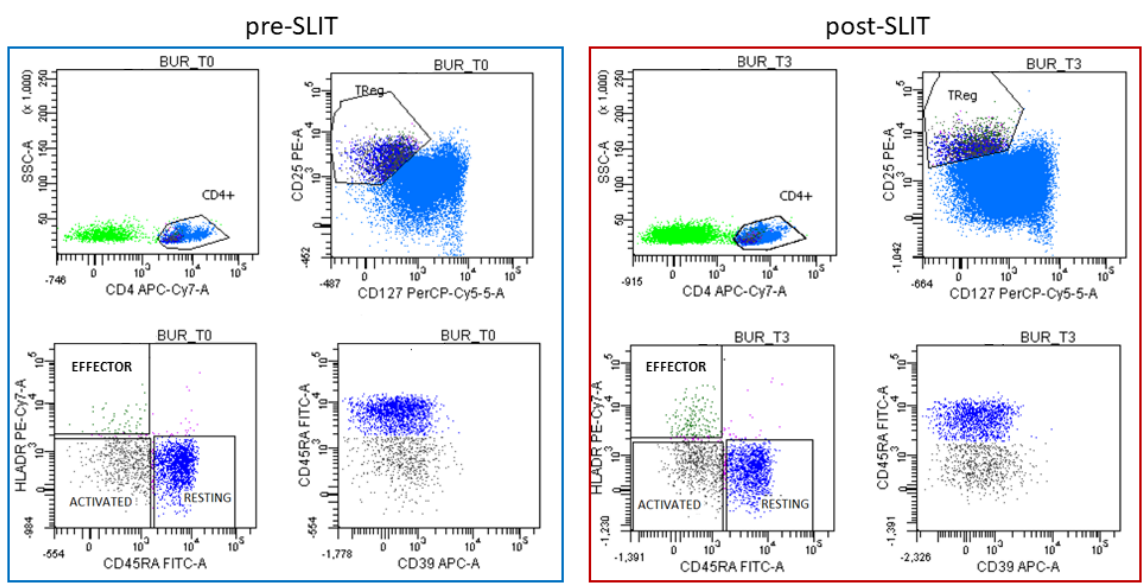


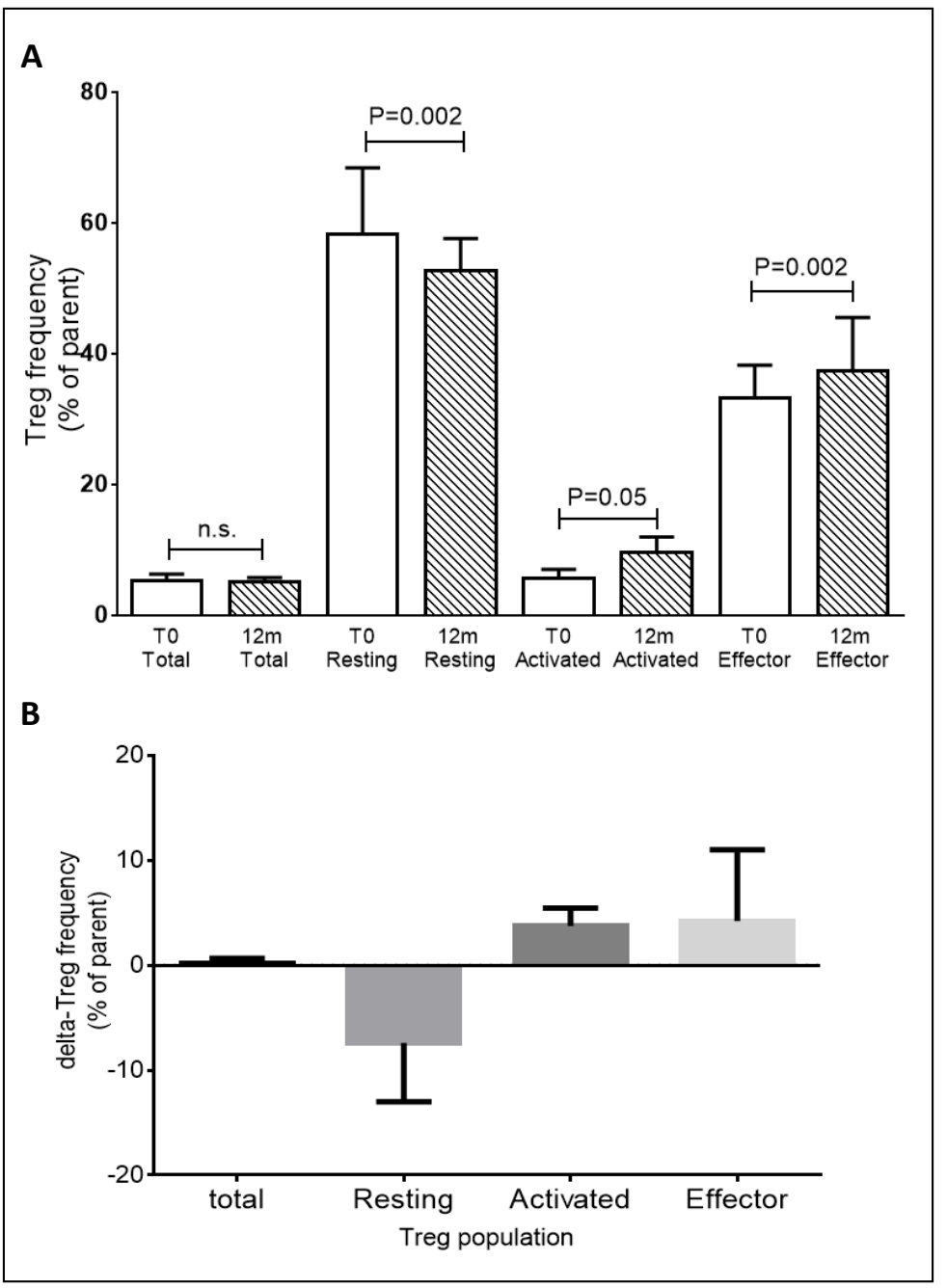




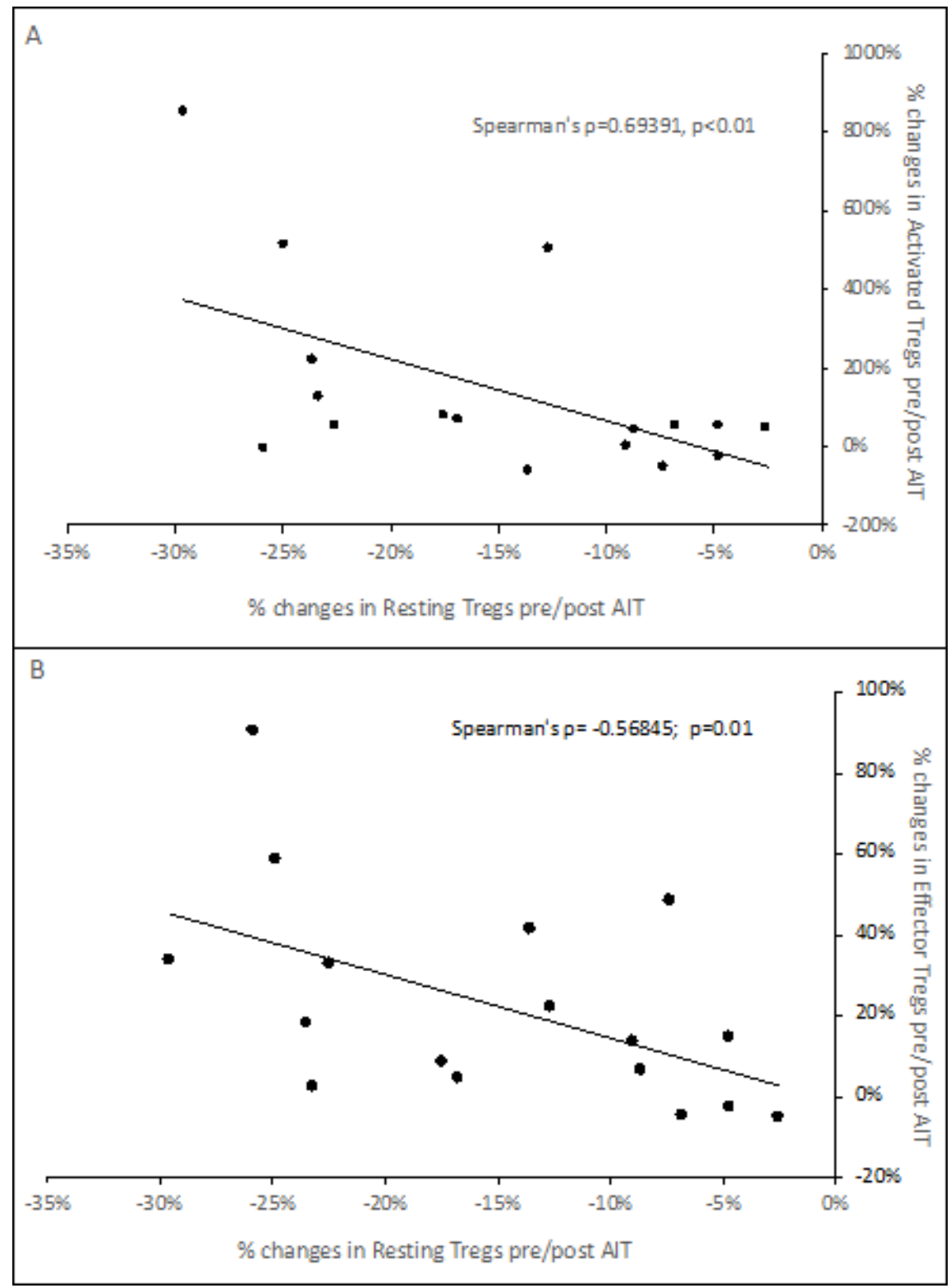



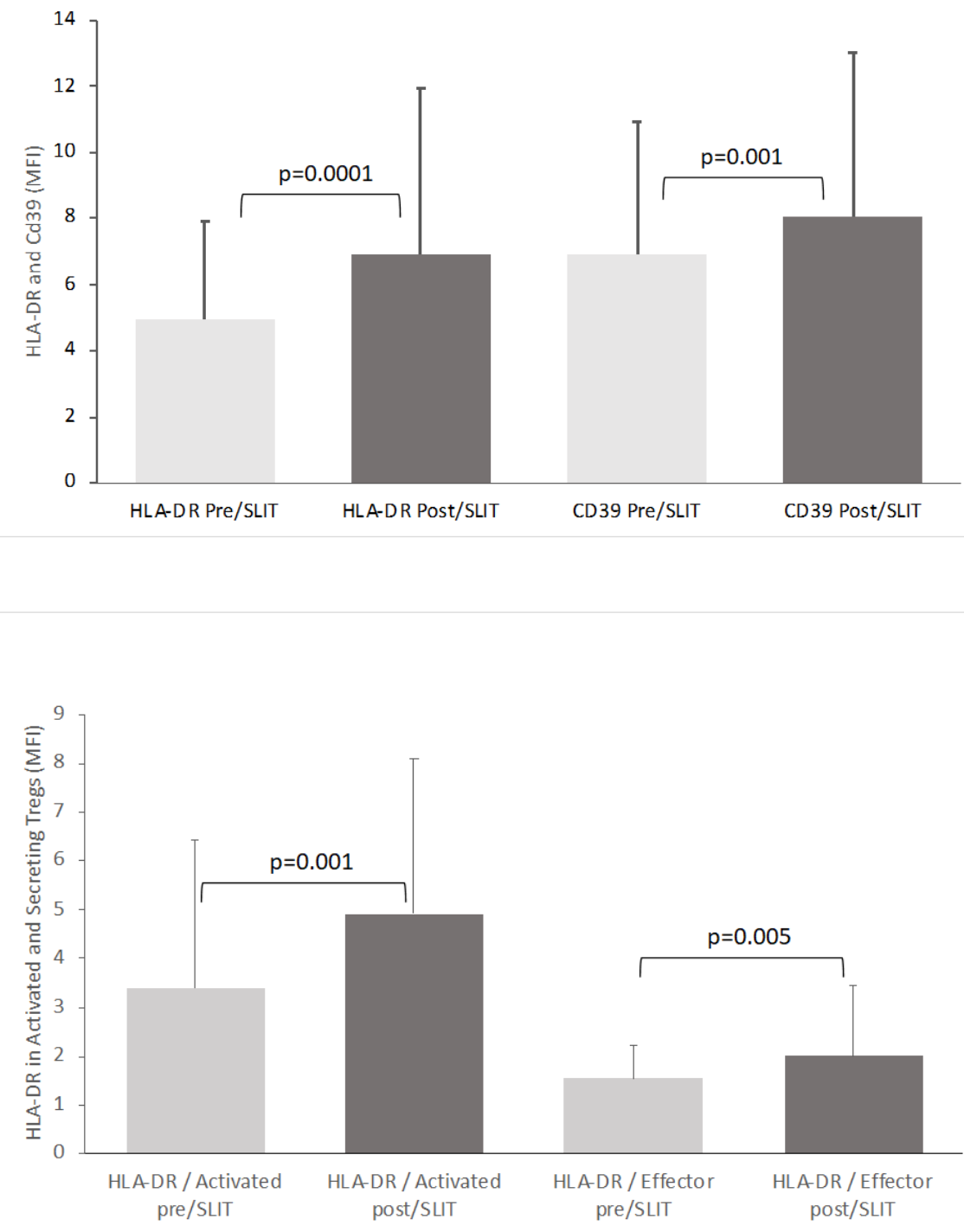


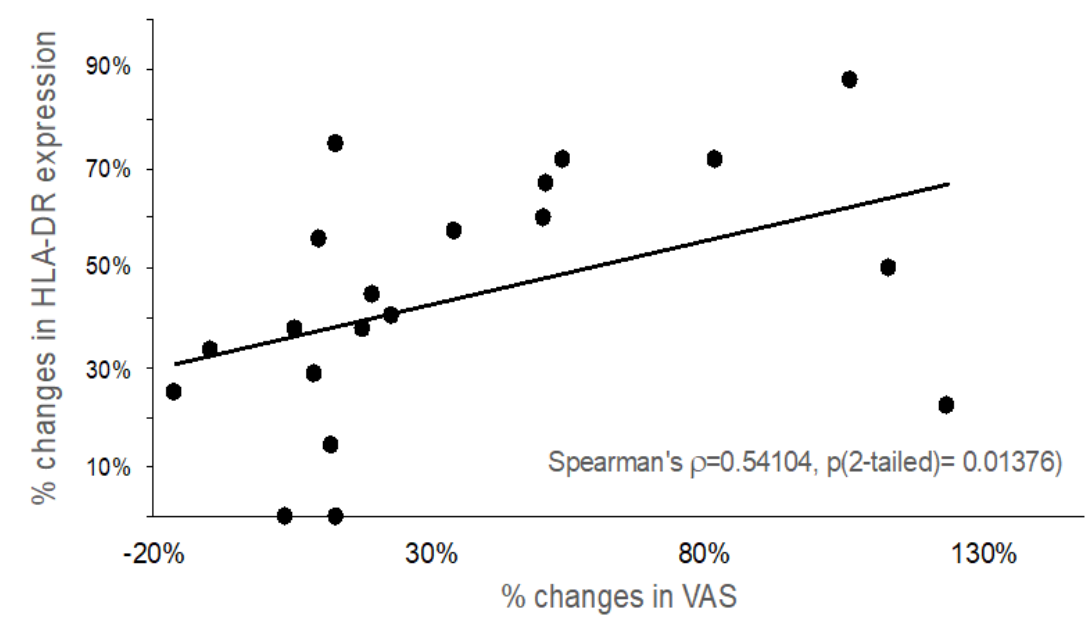




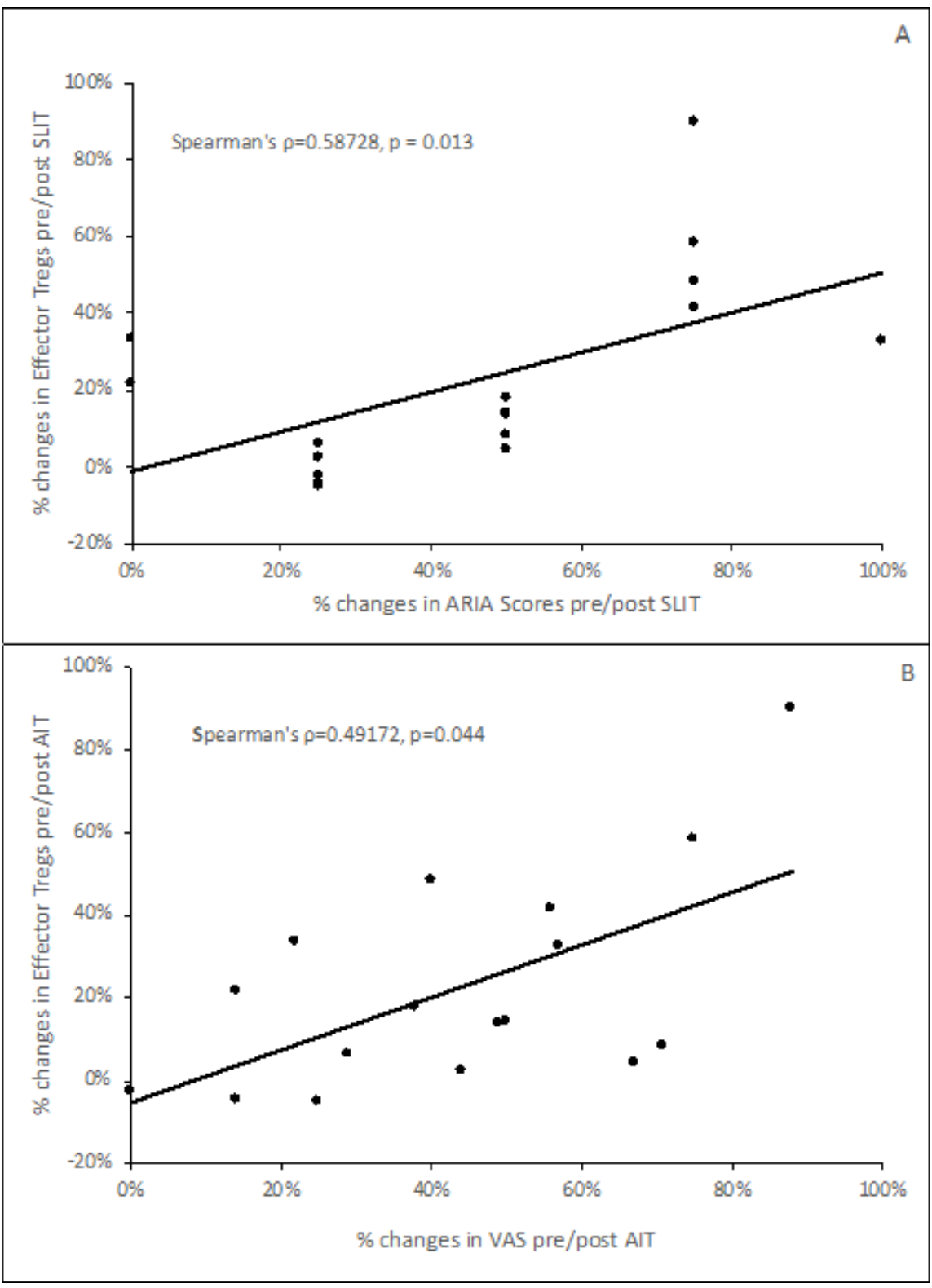

